\title{
Metabolic profiles of adipose-derived and bone marrow-derived stromal cells from elderly coronary heart disease patients by capillary liquid chromatography quadrupole time-of-flight mass spectrometry
}

\author{
JIAN-ZHONG LI ${ }^{1,2^{*}}$, HUI QU ${ }^{1,3^{*}}$, JIAN WU ${ }^{1,4}$, FAN ZHANG $^{5}$, ZHI-BO JIA ${ }^{1}$, JIAN-YONG SUN ${ }^{6}$, \\ BO LV ${ }^{1,4}$, YUE KANG ${ }^{7}$, SHU-LIN JIANG ${ }^{1}$ and KAI KANG ${ }^{1}$ \\ ${ }^{1}$ Key Laboratory of Education of the Ministry for Myocardial Ischemia, Division of Cardiovascular Surgery, \\ The Second Affiliated Hospital of Harbin Medical University, Harbin, Heilongjiang 150086; \\ ${ }^{2}$ Division of Thoracic Surgery, The 211th Hospital of the Chinese People's Liberation Army, Harbin, Heilongiiang 150080; \\ Divisions of ${ }^{3}$ Pediatrics and ${ }^{4}$ Cardiology, The Second Affiliated Hospital of Harbin Medical University, Harbin, \\ Heilongjiang 150086; ${ }^{5}$ Division of Epidemiology and Biostatistics, School of Public Health, Harbin Medical University, \\ Harbin, Heilongjiang 150000; ${ }^{6}$ Division of Thoracic Surgery, Tang Du Hospital, The Fourth Military Medical University, \\ Xi'an, Shaanxi 710032; ${ }^{7}$ Department of General Surgery, The Second Affiliated Hospital of \\ Harbin Medical University, Harbin, Heilongjiang 150000, P.R. China
}

Received April 7, 2017; Accepted October 6, 2017

DOI: $10.3892 / \mathrm{ijmm} .2017 .3198$

\begin{abstract}
Adipose-tissue derived mesenchymal stem cell (ADSC)-based therapy is a promising option for patients with atherosclerotic conditions, including coronary artery disease. However, the potential differences in the metabolic characteristics between bone marrow-derived mesenchymal stem cells (BMSCs) and ADSCs have remained to be fully elucidated. The present study aimed to compare the metabolic
\end{abstract}

Correspondence to: Professor Shu-lin Jiang or Professor Kai Kang, Key Laboratory of Education of the Ministry for Myocardial Ischemia, Division of Cardiovascular Surgery, The Second Affiliated Hospital of Harbin Medical University, 246 Xue Fu Road, Harbin, Heilongjiang 150000, P.R. China

E-mail: jiangshulin111@163.com

E-mail: kangkai1975@sina.com

"Contributed equally

Abbreviations: CHD, coronary heart disease; MSCs, mesenchymal stromal cells; ADSCs, adipose tissue-derived mesenchymal stem cells; BMSCs, bone marrow-derived mesenchymal stem cells; QC, quality control; PCA, principal component analysis; PLS-DA, partial least squares discriminant analysis; $\mathrm{m} / \mathrm{z}$, measured mass to charge ratio; ESI, electron spray ionization; VIP, variable importance in the projection; HMDB, Human Metabolome Database; KEGG, Kyoto Encyclopaedia of Genes and Genomes; CLA, conjugated linoleic acid; PPARs, peroxisome proliferator-activated receptors

Key words: metabolism, bone marrow-derived mesenchymal stem cells, adipose tissue-derived mesenchymal stem cells, conjugated linoleic acid, linoleic acid pathway profiles of BMSCs and ADSCs via liquid chromatography quadrupole time-of-flight mass spectrometry. BMSCs and ADSCs obtained from elderly coronary heart disease patients were cultured, and after three passages, supernatants of each cell type were collected and systematically analysed. Substantial differences were detected between the metabolite signatures of ADSCs and BMSCs. In addition, further analysis using partial least-squares discriminant analysis score plots indicated significant differences between the supernatants of the two cell types. The following metabolites were deemed to be responsible for the potential differences in the metabolic characteristics of BMSCs and ADSCs: D-lactic acid, hydroxyindoleacetaldehyde, $\alpha$-D-glucose, bovinic acid, 9,10-epoxyoctadecenoic acid, glyceraldehyde, phenylpyruvic acid, L-octanoylcarnitine, retinyl ester, $\alpha$-ketoisovaleric acid, guanidoacetic acid, $\mathrm{N}$-acetylneuraminic acid, imidazoleacetic acid riboside, sphingosine and pseudouridine 5'-phosphate. Based on these findings, there may be significant differences in the following metabolic pathways: The linoleic acid metabolic pathway, galactose metabolism, argentines and proline metabolism, retinol metabolism, glycine and serine metabolism, galactose metabolism, and amino sugar and nucleotide sugar metabolism. In conclusion, substantial differences in metabolic characteristics were detected between BMSCs and ADSCs, which may be associated with the different efficacies of atherosclerosis therapies employing these cell types.

\section{Introduction}

Despite significant improvement in diagnosis and treatment strategies in recent years, atherosclerosis and the consequent diseases remain major contributors to mortality and morbidity 
worldwide (1). Pathophysiologically, atherosclerosis is recognized as an inflammatory disease characterized by the activation and migration of inflammatory cells into the subendothelial layer of the arteries. Coronary heart disease (CHD), which is caused by atherosclerotic lesions in the coronary arteries, has become the most important public health problem in developed as well as developing countries, and the incidence is continuously rising worldwide with the acceleration of population aging $(2,3)$. In addition to conventional risk factors that have been associated with atherosclerosis and CHD, overweight and obesity have been linked to the pathogenesis of the above diseases $(4,5)$. With the increasing prevalence of obesity in the global population (6), atherosclerosis-associated diseases are expected to be even more prevalent in the future (7). Therefore, the development of effective therapies against atherosclerosis is of great clinical significance.

Indeed, marked improvements have been made regarding treatment options for atherosclerosis and associated diseases $(8,9)$, and stem cell-based therapies are promising for patients with atherosclerosis, particularly CHD. With their characteristics of extensive proliferation and multipotency, stem cells have been suggested to be effective for repairing of vascular atherosclerotic lesions (7,10-12). Mesenchymal stromal cells (MSCs), which include bone marrow stromal cells (BMSCs) and adipose-tissue derived mesenchymal stem cell (ADSC), are multipotent adult stem cells that are most commonly applied in studies on stem-cell based therapies for atherosclerotic diseases $(13,14)$. In addition to the use of MSCs themselves, bioengineering approaches based on gene therapy using MSCs have also been explored in several preclinical studies (15-17). The benefits of MSC-based therapies in atherosclerosis have been suggested to involve numerous potential mechanisms, including homing of MSCs to atherosclerotic lesions, production of active cytokines, modulation of the immune response, improved endothelial repair and attenuation of thrombosis formation (18-20).

Although BMSCs are the most commonly used type of stem cells in preclinical studies on cell-based therapies for atherosclerosis, the relative rarity of these cells and the invasive procedures required for their harvesting have limited their use. As ADSCs are more readily accessible than BMSCs (21), they are also considered to be a potential cell source for the treatment of atherosclerotic diseases. However, the differences in the biological characteristics of ADSCs and BMSCs remain to be fully elucidated. No significant differences in the morphology and immune phenotype have been identified between BMSCs and ADSCs (22). However, the proliferative activity and apoptotic tolerance of ADSCs were reported to be higher than those of BMSCs (23-25). In addition, the cell population, maximum lifespan and multipotency of BMSCs were found to decrease more rapidly with increasing donor age compared with ADSCs $(26,27)$. MSCs have been demonstrated to be capable of enhancing angiogenesis and improving cardiac function in vivo. Kim et al (28) compared the therapeutic potential of ADSCs and BMSCs by transplanting the same number of cells in a nude mouse model of hind limb ischemia. The results indicated that ADSCs are associated with better blood flow recovery than BMSCs. In a rodent model injected with ADSCs to reconstruct abdominal wall muscle defects, angiogenesis and muscle healing were significantly improved compared with those in animals administered BMSCs (29). In addition, an experimental study demonstrated that ADSCs may induce a greater improvement in infarct area and left ventricle infarct wall thickness than BMSCs (30). The above studies also indicated that application of ADSCs in vivo in ischemic disease was associated with enhanced angiogenesis and a greater improvement in heart function in terms of efficacy and accessibility. The potential mechanisms underlying these differences have not been comprehensively described, and differences in the metabolic characteristics of the two stem cell types may be involved. Therefore, the present study applied liquid chromatography quadrupole time-of-flight mass spectrometry (LC-QTOF-MS) to explore the differences in the metabolites of BMSCs and ADSCs derived from elderly patients with CHD.

\section{Materials and methods}

Patients. A total of 30 elderly patients (age, $\geq 60$ years) with CHD and without hyperlipidemia and/or other metabolic abnormalities who were hospitalized at The Second Affiliated Hospital of Harbin Medical University (Harbin, China) from January, 2015 to October, 2016 were enrolled in the present study. The study protocol was approved by the Ethics Committee of The Second Affiliated Hospital of Harbin Medical University, and informed consent was obtained from all patients.

Cell culture. Bone marrow was collected from $15 \mathrm{CHD}$ patients. The bone marrow was aspirated under local anaesthesia from the sternum and collected in heparinized tubes. Dulbecco's modified Eagle's medium (Gibco; Thermo Fisher Scientific, Inc., Waltham, MA, USA) with $3.7 \mathrm{~g} / 1$ sodium bicarbonate, $1 \%$ penicillin and streptomycin, and $10 \%$ fetal bovine serum (Biological Industries Israel Beit-Haemek, Ltd., Kibbutz Beit-Haemek, Israel) was used for culturing the isolated cells. After $72 \mathrm{~h}$, unattached cells and residual non-adherent red blood cells were removed by washing with phosphate-buffered saline (PBS). ADSCs were derived from adipose tissue of abdominal subcutaneous fat collected under anaesthesia from the other $15 \mathrm{CHD}$ patients as previously described (31). The adipose tissues were washed with PBS containing $1 \%$ penicillin and streptomycin and subsequently digested with collagenase type I $(1 \mathrm{mg} / \mathrm{ml}$; Sigma-Aldrich; Merck KGaA, Darmstadt, Germany) at $37^{\circ} \mathrm{C}$ for $45-60 \mathrm{~min}$ according to the manufacturer's instructions for the collagenase with intermittent shaking. Subsequently, the suspension was filtered using a $200-\mu \mathrm{m}$ nylon mesh and the suspension was then centrifuged at $600 \mathrm{x} \mathrm{g} / \mathrm{min}$ at $4^{\circ} \mathrm{C}$ for $10 \mathrm{~min}$, to separate the floating adipocytes. The cells were then cultured in a humidified atmosphere containing $5 \% \mathrm{CO}_{2}$ at $37^{\circ} \mathrm{C}$ with the medium replaced every 3 days. At passage $3,10^{5}$ cells in $2 \mathrm{ml}$ cell culture medium were seeded in 6-well plates. After 3 days, the supernatants were collected and preserved at $-80^{\circ} \mathrm{C}$ for subsequent analyses.

Sample preparation. Supernatant preparation for the analysis of BMSCs and ADSCs was based on the following procedure: In brief, frozen supernatant samples were thawed at $4^{\circ} \mathrm{C}$ for $50 \mathrm{~min}$. After vortexing for $10 \mathrm{sec}$, the solutions were 
Table I. Clinical characteristics of BMSC and ADSC donors ( $\mathrm{n}=15$ per group).

\begin{tabular}{lccc}
\hline Characteristic & BMSC donors & ADSC donors & P-value \\
\hline No. of subjects & 15 & 15 & - \\
Age, years (median, range) & $64,61-73$ & $65,61-75$ & 0.36 \\
Weight, kg (median, range) & $67,55-83$ & $65,50-85$ & 0.48 \\
Sex & $8 \mathrm{M}, 7 \mathrm{~F}$ & $6 \mathrm{M}, 9 \mathrm{~F}$ & - \\
History of coronary heart disease, years (median, range) & $18,12-25$ & $19,13-26$ & 0.44 \\
Fasting glucose, mmol/l (median, range) & $5.4,4.2-6.1$ & $5.2,4.5-6.1$ & 0.47 \\
\hline
\end{tabular}

BMSC, bone marrow-derived mesenchymal stem cell; ADSC, adipose tissue-derived mesenchymal stem cell; M, male; F, female.

centrifuged at $4,000 \mathrm{xg}$ for $10 \mathrm{~min}$ at $4^{\circ} \mathrm{C}$. The upper aliquot solution $(200 \mu \mathrm{l})$ was transferred to a clean 2 -ml centrifuge tube and then acetonitrile $(1,000 \mu \mathrm{l})$ was added. After vortexing for $2 \mathrm{~min}$, the samples were centrifuged at $12,000 \mathrm{x} \mathrm{g}$ for $15 \mathrm{~min}$ at $4^{\circ} \mathrm{C}$. The upper solution $(1,000 \mu \mathrm{l})$ was transferred to a clean 2-ml centrifuge tube and then evaporated to dryness over a heat block at $35^{\circ} \mathrm{C}$ under nitrogen gas. The residue was dissolved in $200 \mu \mathrm{l}$ acetonitrile/water (1:3, v/v) via vortexing for $1 \mathrm{~min}$ and centrifugation at $12,000 \mathrm{x}$ g for $15 \mathrm{~min}$ at $4^{\circ} \mathrm{C}$. The supernatant $(200 \mu \mathrm{l})$ was transferred to an autosampler vial and injected into the LC-QTOF-MS (6530 series; Agilent Technologies, Inc., Santa Clara, CA, USA) apparatus for analysis. Equal amounts of supernatant samples from 15 ADSC cultures and 15 BMSC cultures as the samples were mixed for quality control (QC).

Chromatography. Each 10- $\mu 1$ aliquot of sample was injected into a $2.1 \times 100 \mathrm{~mm}(1.8 \mathrm{~mm})$ ZORBAX SB-C18 column for subsequent rapid resolution liquid chromatography (6530 series) (both from Agilent Technologies, Inc.). A mixture of acetonitrile containing $0.1 \%$ formic acid (phase A) and water containing $0.1 \%$ formic acid (phase B) were used as the mobile phase for electron spray ionisation in positive mode $\left(\mathrm{ESI}^{+}\right)$, while a mixture of acetonitrile (phase $\mathrm{A}$ ) and water (phase B) was used as the mobile phase for ESI in negative mode $\left(\mathrm{ESI}^{-}\right)$. The protocols for the linear mobile phase gradient were as follows: $95 \%$ A held for $1 \mathrm{~min}$; decreased to $2 \%$ A by $10 \mathrm{~min}$; held at $2 \%$ A until $13 \mathrm{~min}$; increased to 95\% A by $13.1 \mathrm{~min}$; and held at $95 \%$ A until $20 \mathrm{~min}$. The flow rate of the mobile phase was $0.3 \mathrm{ml} / \mathrm{min}$ at $40^{\circ} \mathrm{C}$.

MS. MS was performed using an Agilent 6530-QTOF MS apparatus (6530 series; Agilent Technologies, Inc.) operating in $\mathrm{ESI}^{+}$or $\mathrm{ESI}^{-}$mode. The capillary voltage was set as $4.0 \mathrm{kV}$ for $\mathrm{ESI}^{+}$and $3.5 \mathrm{kV}$ for $\mathrm{ESI}^{-}$. Nitrogen was applied as the desolvation gas at a flow rate of $10 \mathrm{l} / \mathrm{min}$. The desolvation temperature was $350^{\circ} \mathrm{C}$. The centroid data were obtained with the full scan mode [mass-to-charge ratio $(\mathrm{m} / \mathrm{z})=50-1,000]$.

Data pre-processing and annotation. The raw data were converted into mzData-format files using MassHunter Qualitative Analysis Software (v. B.04.00; Agilent Technologies, Inc.) and these files were further imported to the XCMS package in $\mathrm{R}$ (v. 3.0.2) (r-project.org/) for pre-processing. The analyses followed the default XCMS parameter settings, with the following exceptions: xcms Set (fwhm, 10), group (minfrac, 0.5; bw, 30) and rector (method, 'obiwarp'). The definitions are as follows: fwhm, specifying the full width at half maximum of matched filtration Gaussian model peak; minfrac, defining the minimum fraction of samples in at least one sample group in which the peaks have to be present to be considered as a peak group; and bw, defining the bandwidth (standard deviation of the smoothing kernel) to be used.

Subsequently, a data matrix was generated, including results of retention time, $\mathrm{m} / \mathrm{z}$ values and peak intensity. CAMERA in R (v. 3.0.2) was used to annotate isotope peaks and generate adducts and fragments in the peak lists (32). A total of 1,668 ions in $\mathrm{ESI}^{+}$mode and 829 ions in $\mathrm{ESI}^{-}$mode were included for subsequent statistical analysis.

Statistical analysis. First, principal component analysis (PCA) was used to detect the grouping trends and outliers (33). The Wilcoxon rank sum test was then applied to determine the significance of each metabolite at $\mathrm{P}<0.05$. To identify the differences in metabolites between BMSCs and ADSCs, a partial least squares discriminant analysis (PLS-DA) was used (33). Permutation tests with 100 iterations were included to validate the supervised model and avoid overfitting (34). Based on the PLS-DA model, parameters that described the variable importance in the projection (VIP) for each metabolite were calculated. With thresholds of P-values and VIP values of 0.05 and 1 , respectively, the metabolic biomarkers were detected. The Wilcoxon rank sum test was used on the R platform (v. 3.0.2). The PCA and PLS-DA were performed using SIMCA-P (v. 11.5; Umetrics, Malmö, Sweden).

\section{Results}

PCA score plots for discriminating BMSCs and ADSCs. The baseline characteristics of the donors are presented in Table I. There were no significant differences between the groups of donors (BMSC donors: 8 males and 7 females; median age, 64 years; age range, 61-73 years; median weight, $67 \mathrm{~kg}$; weight range, $55-83 \mathrm{~kg}$; mean fasting glucose, $5.4 \mathrm{mmol} / \mathrm{l}$; and fasting glucose range, 4.2-6.1 mmol/1. ADSC donors: 6 males and 9 females; median age, 65 years; age range, 61-75 years; median weight, $65 \mathrm{~kg}$; weight range, $50-85 \mathrm{~kg}$; mean fasting glucose, $5.2 \mathrm{mmol} / \mathrm{l}$; fasting glucose range, 4.5-6.1 mmol/1). Metabolic analysis revealed numerous metabolic differences between BMSCs and ADSCs. The results 

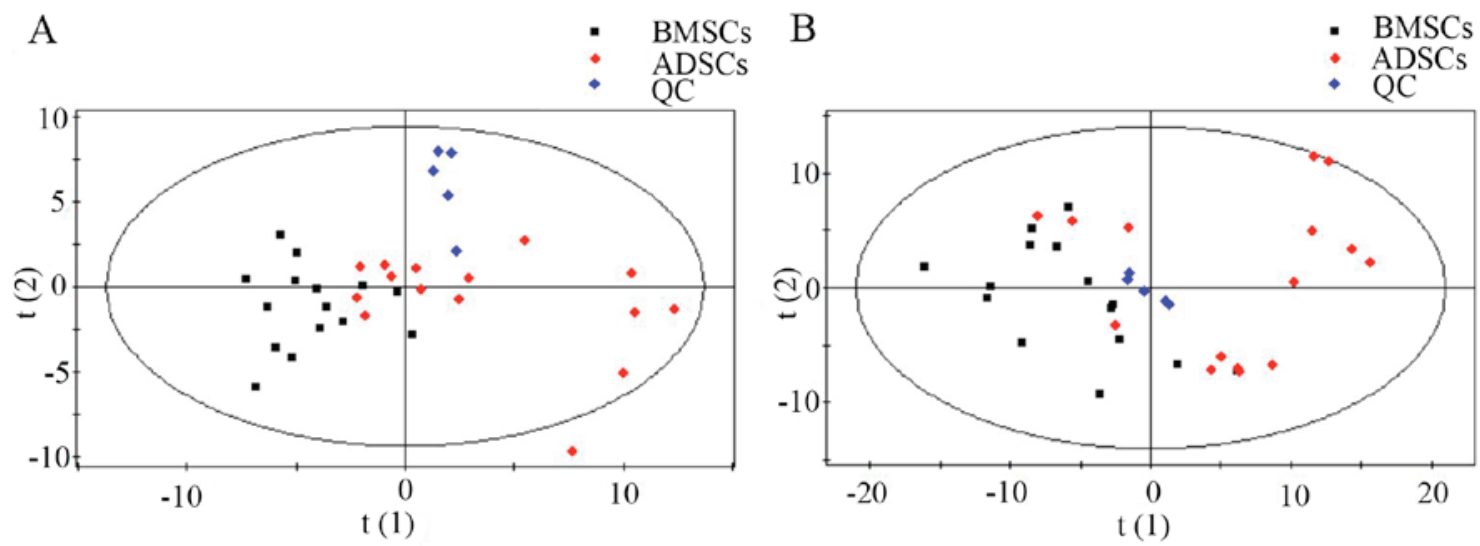

Figure 1. Principal component analysis score plots for discriminating BMSCs and ADSCs in ESI ${ }^{+}$and ESI- modes. BMSCs, bone marrow-derived mesenchymal stem cells; ADSCs, adipose tissue-derived mesenchymal stem cells; ESI, electron spray ionization; QC, quality control.
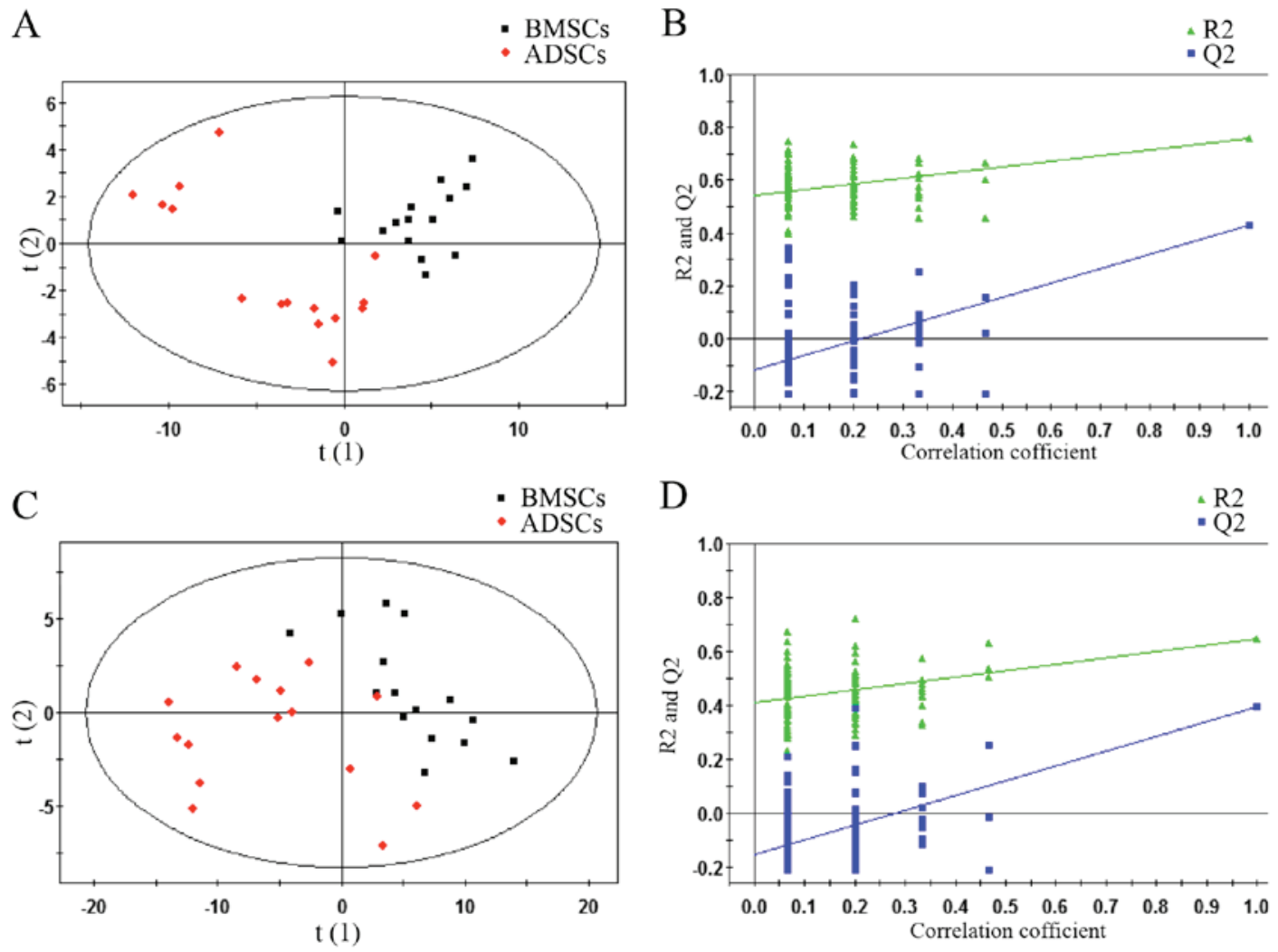

Figure 2. PLS-DA plots and validation plots for discriminating ADSCs and BMSCs in ESI ${ }^{+}$and ESI- modes. (A) PLS-DA plot in ESI ${ }^{+}$mode; (B) validation plot in ESI ${ }^{+}$mode; (C) PLS-DA plot in ESI- mode; (D) validation plot in ESI- mode. BMSCs, bone marrow-derived mesenchymal stem cells; PLS-DA, partial least squares discriminant analysis score; $\mathrm{ESI}^{+}$, electron spray ionisation in positive; ESI- electron spray ionisation in negative; ADSCs, adipose tissue-derived mesenchymal stem cells; ESI, electron spray ionization; Q2, second quartile; $\mathrm{R}^{2}$, coefficient of determination; PLS-DA, partial least squares discriminant analysis score; horizontal axis t, principal component one; vertical axis t, principal component two.

of the overall PCA based on all the samples suggested that the QC samples were closely clustered in plots of PCA scores, which demonstrated that the results of the metabolic profiling platform were robust. In addition, no outliers were present on the whole, and separation trends were observed between BMSCs and ADSCs (Fig. 1).

PLS-DA plots and validation plots for discriminating BMSCs and ADSCs. Via the application of the $\mathrm{ESI}^{+}$and $\mathrm{ESI}^{-}$modes, all of the statistically significant ions were analysed $(\mathrm{P}<0.05$ and VIP>1) (Fig. 2). Subsequently, a supervised PLS-DA model was used to identify differences between BMSCs and ADSCs. As presented in the PLS-DA score plot, an obvious separation between BMSCs and ADSCs was present in the $\mathrm{ESI}^{+}$mode (Fig. 2A) and ESI mode (Fig. 2C). The PLS-DA models contained two predictive components in $\mathrm{ESI}^{+}$mode $\left[\mathrm{R}^{2} \mathrm{X}=0.409 ; \mathrm{R}^{2} \mathrm{Ycum}=0.759\right.$; cumulative second quartile $\left(\mathrm{Q}^{2}\right.$ cum $\left.)=0.429\right]$ and two components in $\mathrm{ESI}^{-}$mode $\left(\mathrm{R}^{2} \mathrm{X}=0.55\right.$; $\mathrm{R}^{2}$ Ycum $=0.647 ; \mathrm{Q}^{2}$ cum $=0.398$ ). Permutation tests including 100 iterations and containing two predictive components were 
Table II. Detailed information on 16 supernatant metabolites.

$\mathrm{A}, \mathrm{ESI}^{+}$mode

\begin{tabular}{|c|c|c|c|c|c|c|c|c|}
\hline ID & Metabolite & $\mathrm{m} / \mathrm{z}$ & $\mathrm{RT}(\min )$ & ppm & $\mathrm{FC}^{\mathrm{a}}$ & P-value & VIP & Pathway \\
\hline $\mathrm{P} 1$ & Glyceraldehyde & 113.0197 & 56.58 & 10 & 0.79 & 0.036203 & 1.299 & Glycerolipid metabolism \\
\hline $\mathrm{P} 2$ & $\begin{array}{l}\text { Pyrroline } \\
\text { hydroxycarboxylic acid }\end{array}$ & 130.0505 & 56.52 & 5 & 1.21 & 0.012093 & 1.8038 & $\begin{array}{l}\text { Arginine and proline } \\
\text { metabolism }\end{array}$ \\
\hline $\mathrm{P} 3$ & Phenylpyruvic acid & 165.0547 & 56.58 & 0 & 0.95 & 0.044253 & 1.0174 & $\begin{array}{l}\text { Phenylalanine and tyrosine } \\
\text { metabolism }\end{array}$ \\
\hline P4 & $\begin{array}{l}\text { Imidazoleacetic } \\
\text { acid riboside }\end{array}$ & 281.0754 & 56.1 & 3 & 1.40 & 0.019103 & 1.4403 & Histidine metabolism \\
\hline P5 & L-octanoylcarnitine & 288.217 & 505.38 & 0 & 0.68 & 0.023787 & 1.4542 & $\begin{array}{l}\text { Mitochondrial } \beta \text {-oxidation } \\
\text { of short chain saturated } \\
\text { fatty acids }\end{array}$ \\
\hline P6 & Sphingosine & 322.2682 & 840.22 & 10 & 1.39 & 0.019103 & 1.6411 & Sphingolipid metabolism \\
\hline P7 & $\begin{array}{l}\text { Pseudouridine } \\
5 \text { '-phosphate }\end{array}$ & 325.0374 & 56.3 & 17 & 1.47 & 0.048815 & 1.4483 & Pyrimidine metabolism \\
\hline P8 & Retinyl ester & 325.2118 & 870.735 & 5 & 0.74 & 0.040057 & 1.7041 & Retinol metabolism \\
\hline
\end{tabular}

$\mathrm{B}, \mathrm{ESI}^{-}$mode

\begin{tabular}{|c|c|c|c|c|c|c|c|c|}
\hline N1 & D-Lactic acid & 89.02636 & 54.65 & 21 & 0.77 & 0.009531 & 1.4873 & Pyruvate metabolism \\
\hline $\mathrm{N} 2$ & $\alpha$-ketoisovaleric acid & 115.0404 & 59.73 & 2 & 1.37 & 0.015247 & 1.4307 & $\begin{array}{l}\text { Pantothenate and } \\
\text { CoA biosynthesis }\end{array}$ \\
\hline N3 & Guanidoacetic acid & 116.044 & 59.7 & 9 & 1.36 & 0.015247 & 1.4643 & $\begin{array}{l}\text { Glycine and serine } \\
\text { metabolism }\end{array}$ \\
\hline N4 & Hydroxyindoleacetaldehyde & 174.0551 & 465.62 & 5 & 0.76 & 0.040057 & 1.0804 & Tryptophan metabolism \\
\hline N5 & $\alpha$-D-glucose & 179.0563 & 51.34 & 0 & 0.86 & 0.026482 & 1.3418 & Galactose metabolism \\
\hline N6 & Bovinic acid & 279.2292 & 887.24 & 13 & 0.72 & 0.004494 & 1.641 & $\begin{array}{l}\text { Linoleic acid metabolic } \\
\text { pathway }\end{array}$ \\
\hline N7 & 9,10-Epoxyoctadecenoic acid & 295.2244 & 722.755 & 11 & 0.76 & 0.002637 & 1.349 & $\begin{array}{l}\text { Linoleic acid metabolic } \\
\text { pathway }\end{array}$ \\
\hline N8 & $\mathrm{N}$-acetylneuraminic acid & 308.0994 & 51.19 & 2 & 1.55 & 0.044253 & 1.1037 & Amino sugar metabolism \\
\hline N9 & 11,13-EpOME & 295.2244 & 722.755 & 11 & 0.76 & 0.002637 & 1.349 & $\begin{array}{l}\text { Linoleic acid metabolic } \\
\text { pathway }\end{array}$ \\
\hline
\end{tabular}

${ }^{\mathrm{a}} \mathrm{FC}$ was calculated based on means of BMSCs and ADSCs. FC $>1$ means that the biomarker increased in BMSCs compared with that in ADSCs. FC, fold change; RT, retention time; ppm, parts per million; $\mathrm{m} / \mathrm{z}$, mass to charge ratio; VIP, variable importance in the projection; ESI, electron spray ionization; $\mathrm{P}$, positive; $\mathrm{N}$, negative; BMSCs, bone marrow-derived mesenchymal stem cells; ADSCs, adipose tissue-derived mesenchymal stem cells.

also performed (35). The results indicated that the permuted $\mathrm{Q}^{2}$ cum values were lower than the original values in almost all cases (Fig. 2B and D), which further confirmed the validity of the supervised models. $\mathrm{R}^{2}$ identified the outfit of the PLS model. $\mathrm{Q}^{2}$ cum refers to the predicting ability of the PLS model.

Metabolite profiles of potential biomarkers differing between $B M S C s$ and ADSCs. Analysis of VIP values revealed discriminatory metabolites that contributed to the differences between BMSCs and ADSCs. Based on false discovery rate and VIP thresholds of 0.05 and 1 , respectively, differential ions were selected as biomarker candidates for subsequent metabolite identification. The identification procedures were similar to strategies previously published by our group $(36,37)$. In total, 8 metabolites in $\mathrm{ESI}^{+}$mode and 8 metabolites in $\mathrm{ESI}^{-}$mode were identified (Table II). D-lactic acid, hydroxyindoleacetaldehyde, $\alpha$-D-glucose, bovinic acid, 9,10-epoxyoctadecenoic acid, glyceraldehyde, phenylpyruvic 

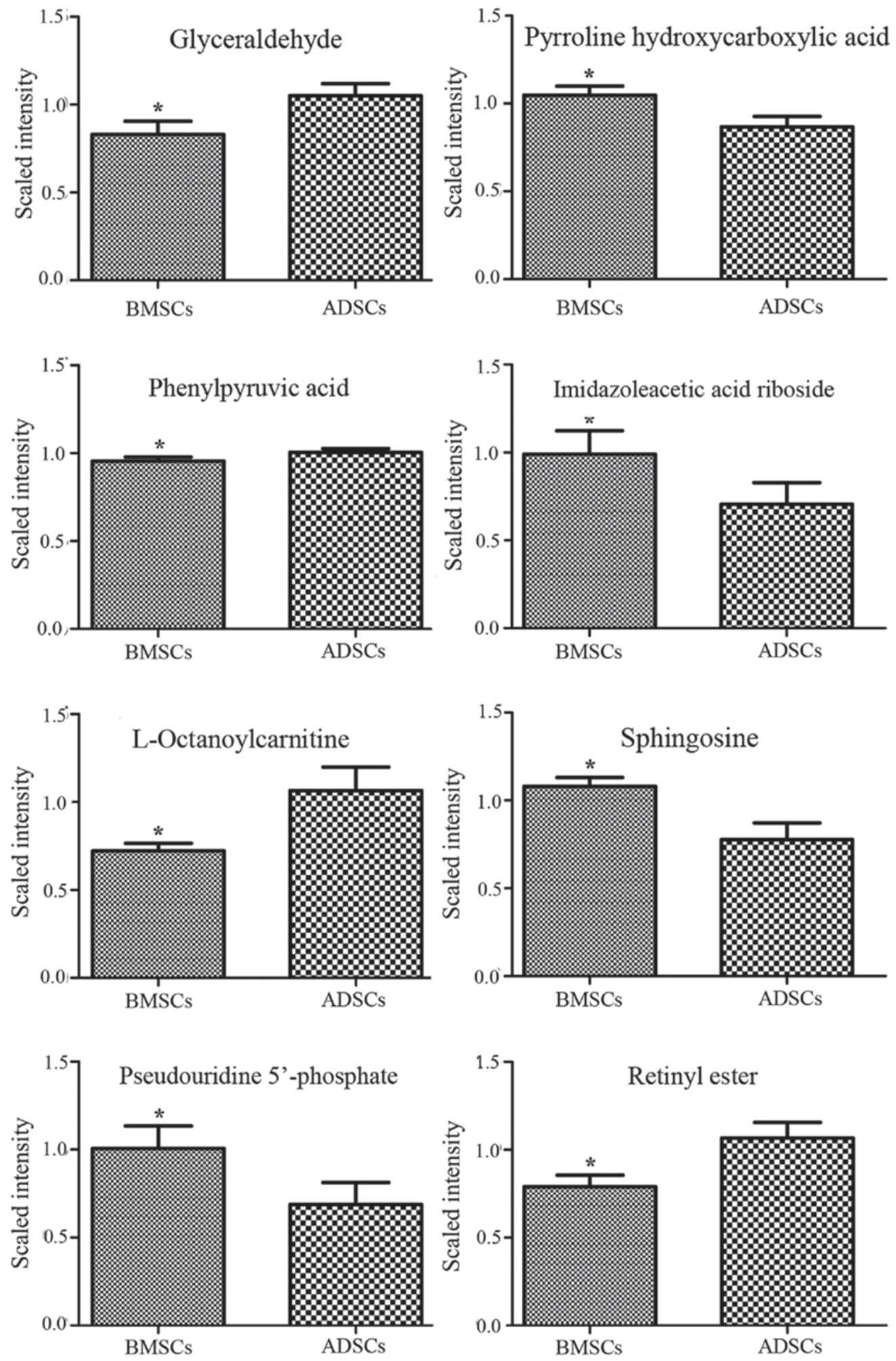

Figure 3. Metabolite profiles of potential biomarkers differing between epithelial BMSCs and ADSCs in positive electron spray ionization mode. Values are expressed as the mean \pm standard error of the mean. ${ }^{*}<0.05$ vs. ADSCs. BMSCs, bone marrow-derived mesenchymal stem cells; ADSCs, adipose tissue-derived mesenchymal stem cells.

acid, L-octanoylcarnitine and retinyl ester were observed to be elevated in the supernatant of ADSCs compared with that of BMSCs (Figs. 3-5). By contrast, $\alpha$-ketoisovaleric acid, guanidoacetic acid, $\mathrm{N}$-acetylneuraminic acid, imidazoleacetic acid riboside, sphingosine and pseudouridine 5'-phosphate levels were lower in the supernatant of ADSCs compared with that of BMSCs (Figs. 3-5). The involved biochemical pathways mapped in the Human Metabolome Database (HMDB) (38) and the Kyoto Encyclopaedia of Genes and Genomes (KEGG) (39) included the linoleic acid metabolic pathway, glycerolipid metabolism, arginine and proline metabolism, mitochondrial $\beta$-oxidation of short chain saturated fatty acids, pyrimidine metabolism, glycine and serine metabolism, galactose metabolism and amino sugar metabolism. 

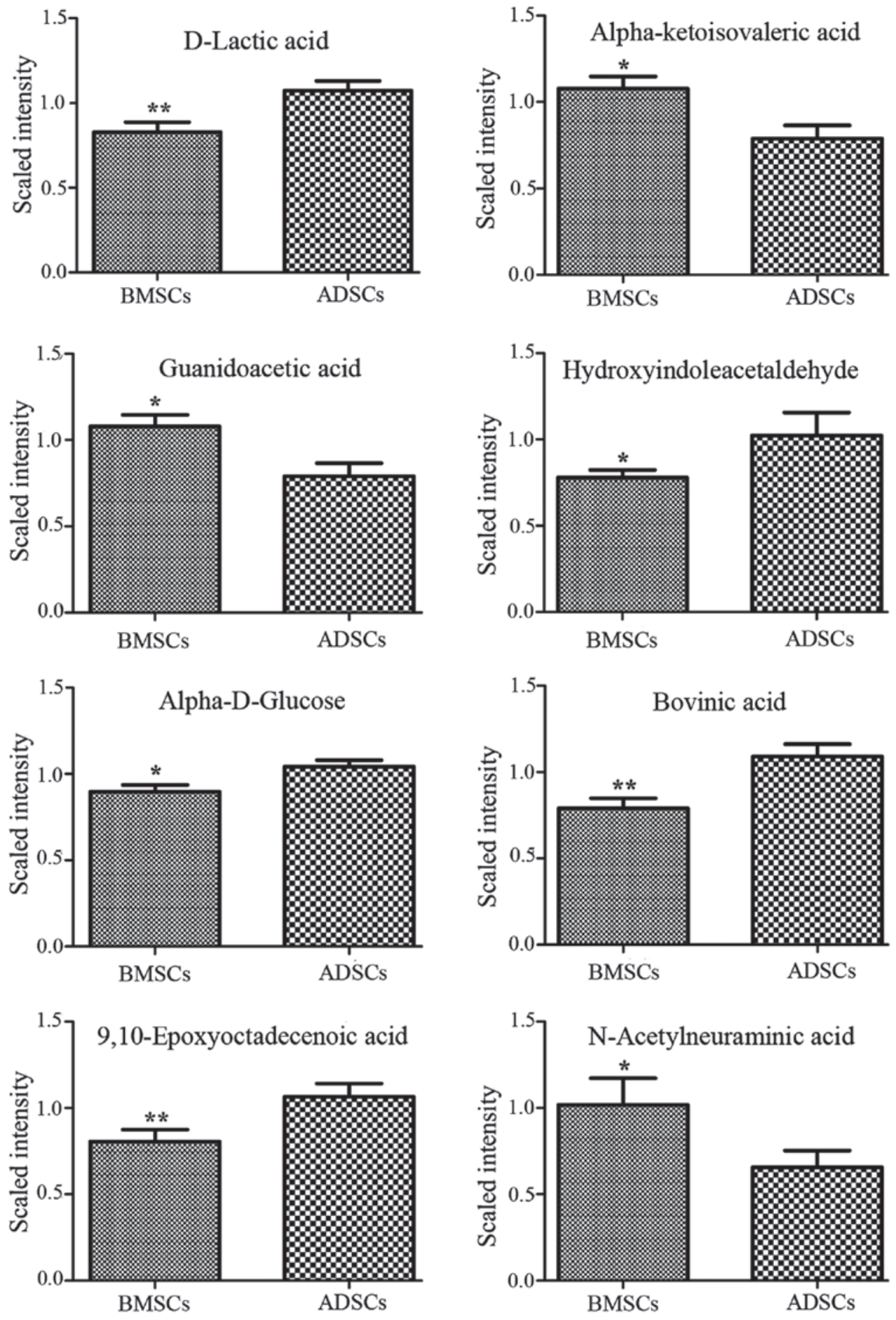

Figure 4. Metabolite profiles of potential biomarkers differing between epithelial BMSCs and ADSCs in negative electron spray ionization mode. Values are expressed as the mean \pm standard error of the mean. ${ }^{*} \mathrm{P}<0.05$ and ${ }^{* *} \mathrm{P}<0.01$ vs. ADSCs. BMSCs, bone marrow-derived mesenchymal stem cells; ADSCs, adipose tissue-derived mesenchymal stem cells.

\section{Discussion}

The results of the Scandinavian Simvastatin Survival Study were published in The Lancet 20 years ago (40). At present, dyslipidemia is major cause of atherosclerotic vascular disease. Recent studies further underlined the significance of dyslipidemia in cardiovascular disease through performing research on lipids [high-density lipoprotein cholesterol (HDL-C), low-density lipoprotein cholesterol (LDL-C) and triglycerides] and cardiovascular disease (41-43). Autologous MSC transplantation has emerged as a novel treatment for atherosclerosis-associated diseases, and pilot studies have demonstrated a promising clinical effect for this treatment strategy. However, the relative efficacies of BMSC- and ADSC-based cellular therapies for atherosclerosis-associated diseases have remained largely elusive. Establishing the metabolic signatures of these cell types will be helpful for understanding differences between them and be of significance for the development of clinical treatments. The present results regarding unknown and annotated analytes indicated that the supernatant of ADSCs contained 


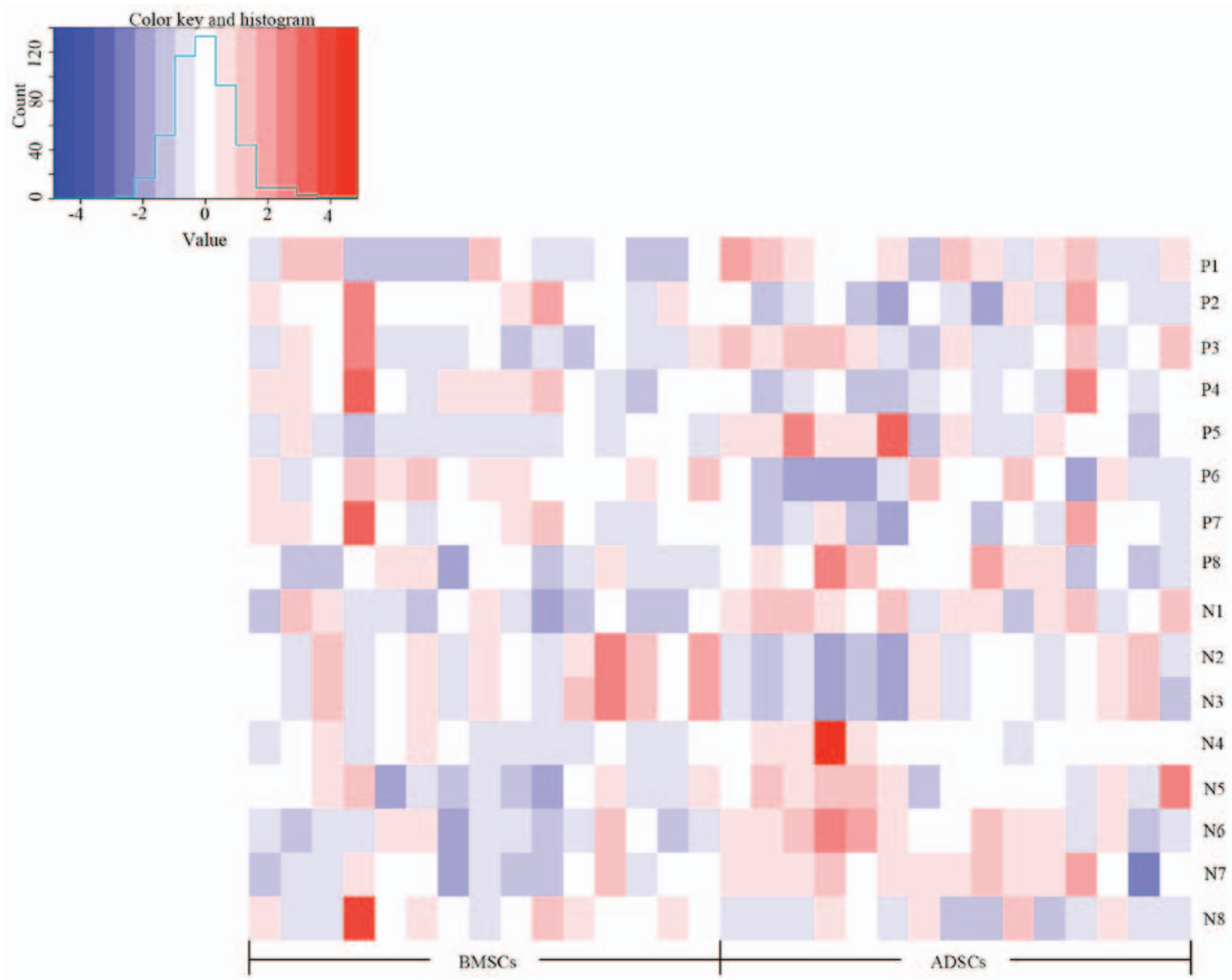

Figure 5. Heat map demonstrating dynamic changes in BMSC and ADSC biomarkers. P1/N1, metabolites detected in positive/negative electron spray ionization mode according to Table II; BMSCs, bone marrow-derived mesenchymal stem cells; ADSCs, adipose tissue-derived mesenchymal stem cells.

significantly different levels of metabolites compared with BMSCs. Of note, the metabolites accounting for the differences between the supernatants of ADSCs and BMSCs were matched with known human metabolites in the HMDB (hmdb.ca/) or KEGG (kegg.jp/kegg/pathway.html), and these results were further confirmed by a manual search for similarities between the annotated and the library spectra for each metabolite.

Overall, the results of the present metabolite pathway enrichment analysis retrieved 15 potential pathways that were considered to be different between ADSCs and BMSCs. Two annotated metabolites included bovinic acid and 9,10-epoxyoctadecenoic acid, which are components of the linoleic acid pathway. The linoleic acid pathway contains 15 metabolites and participates in protecting the body against disease states such as atherosclerosis, thrombosis, diabetes, high blood pressure, skin inflammation, aging and cancer. Bovinic acid is a predominant conjugated linoleic acid (CLA) in human adipose tissue, comprising a group of fatty acids with 18 carbon atoms, and has anti-atherogenic and anticarcinogenic activities $(44,45)$. As the pathophysiological process of atherosclerosis is complex and involves numerous cellular pathways, reversal of particular pathways may not be sufficient for the prevention of the disease. However, studies suggested that administration of CLA may be associated with the regression of atherosclerosis in rabbits (46) and other animal models (47). Evidence from a patient study has demonstrated that CLA has anti-inflammatory effects via the reduction of oxidative stress (48).

Several studies have also demonstrated that consumption of CLA reduced the fat mass or the percentage of body fat in healthy and in obese/overweight adults (49-51). As such, the conclusions from meta-analyses of previous patient studies were that intake of CLA reduced body weight and body-fat mass (52). The potential mechanisms of action of CLA may involve metabolic effects of inhibiting lipogenesis and accelerating lipolysis (53). Via interactions with the peroxisome proliferator-activated receptors (PPARs), CLA has been proven to initiate the transcription of genes associated with the differentiation of adipocytes, which involve lipolysis ( $\beta$-oxidation) and mitochondrial biogenesis (54). Of note, the activation of PPAR $\gamma$ was associated with delayed progression of atherosclerosis and dyslipidemia. In addition, a recent study confirmed that the effects of CLA against inflammation were mainly mediated via the inhibition of nuclear factor- $\mathrm{\kappa B}$ and mitogen-activated protein kinase signalling pathways (55).

Furthermore, clinical studies have reported that CLA may provide a great benefit for human health. An inverse association between cis-9, trans-11 CLA and the risk of myocardial infarction has been detected among Costa Rican subjects (56). Another human study drew a similar conclusion, 
namely that intake of CLA increased HDL-C and reduced the LDL-C/HDL-C ratio in type 2 diabetic patients (57). In addition, CLA was also reported to improve insulin sensitivity in young patients, which was correlated with decreased fasting insulin levels (58). A clinical trial indicated an effect of CLA on Crohn's disease, where intake of $6 \mathrm{~g}$ CLA/day for 12 weeks improved inflammatory bowel disease questionnaire responses and decreased the Crohn's disease activity index (59). In the Swedish Mammography Cohort study, intake of CLA was demonstrated to reduce the risk of colorectal cancer by $13 \%$ and the risk of distal colon cancer by $34 \%$ (60). In a study on breast cancer patients, CLA inhibited tumour metastasis in premenopausal women $(61,62)$. In South African children, the potential preventive effects of CLA on laryngeal papillomatosis have been reported, which may cause airway obstruction in young children (63). The abovementioned patient studies indicated the potential application of CLA in cardiovascular diseases, metabolic syndrome, immune system diseases and cancer, either alone or complementary to present treatments.

BMSCs have been proposed as a cell source for atherosclerosis therapy. However, ADSCs have emerged as a novel cell source with easy accessibility, and they may be collected from elderly patients with less injury than bone marrow. In addition, in elderly patients, BMSCs reside in the bone marrow stroma in smaller quantities compared with those in young patients, whereas the amount of ADSCs is often greater due to the dramatic increase in the incidence of obesity worldwide. Both cell types are well tolerated by humans. However, the relative efficacies of BMSC- and ADSC-based stem cell therapies for patients with atherosclerosis-associated diseases, such as CHD, remain to be determined. A recent study suggested that ADSC transfusion was associated with a repressed increase in body weight and improved dyslipidemia in obese mice (64). In addition, CLA has been proven to stimulate lipolysis in human adipocytes and diminish the synthesis of fatty acids, although the specific mechanisms remain to be determined (65). Furthermore, ADSCs have been suggested to be more immunosuppressive than BMSCs, as ADSCs are associated with a more marked inhibition of the expression of functionally important co-stimulatory molecules on the surface of monocyte-derived dendritic cells (66). The results of the present study suggested that ADSCs may possibly act upon adipose tissue via the production of CLA and participate in the linoleic acid pathway, which may provide additional treatment effects as compared with BMSCs.

Nevertheless, there are some limitations of the present study. The study enrolled 30 patients, all of which were elderly, and used the BMSCs from 15 of them and the ADSCs from the other 15 patients. However, it may have been appropriate to assess the ADSCs and BMSCs from the same patient and then determine the differences in metabolites. Therefore, based on the study design, it cannot be excluded that the differences in metabolites between ADSCs and BMSCs may have been due to them being taken from two different populations/groups. Furthermore, no control group was used, such as a group of younger patients for comparison.

In conclusion, the results of the present study revealed a marked difference regarding the metabolic characteristics of ADSCs and BMSCs. ADSCs exhibited differences regarding components of the linoleic acid pathway, including bovinic acid, 12,13-EpOME, 13-hydroxyoctadecadienoic acid and 9,10-epoxyoctadecenoic acid as compared with BMSCs. These results enhanced the current understanding of the metabolic differences between ADSCs and BMSCs and may represent the underlying mechanisms responsible for the different efficacies of ADSC- and BMSC-based stem cell therapies for atherosclerosis-associated diseases.

\section{Acknowledgements}

This study was supported by the National Natural Science Foundation of China (grant no. 81471805), Chinese Postdoctoral Science Foundation (grant no. 2014M551272), Postdoctoral Science Foundation of Heilongjiang Province (grant no. LBH-Z14135), Scientific Research Project of Educational Department of Heilongjiang Province (grant no. 12541434), Merit Aid Program for Returnees of Human Resource Department of Heilongjiang Province (2014; grant no. 454), Harbin Municipal Science and Technology Research Fund of Innovative Talents Project (grant no. RC2016QN004036), 'Yu Weihan' Outstanding Young Investigator Award (2014) and Earl Bakken Scholarship (2016) to K.K. The authors would like to thank Ms. Li Ruiting (Key Laboratory of Drug Quality Control and Pharmacovigilance, China Pharmaceutical University, Ministry of Education, Nanjing, China) and Ms. Sun Meng (Key Laboratory of Education of the Ministry for Myocardial Ischemia, The Second Affiliated Hospital of Harbin Medical University, Harbin, China) for technical and scientific advice.

\section{References}

1. Go AS, Mozaffarian D, Roger VL, Benjamin EJ, Berry JD, Blaha MJ, Dai S, Ford ES, Fox CS, Franco S, et al; American Heart Association Statistics Committee and Stroke Statistics Subcommittee: Executive summary: heart disease and stroke statistics - 2014 update: a report from the American Heart Association. Circulation 129: 399-410, 2014.

2. Kelly BB, Narula J and Fuster V: Recognizing global burden of cardiovascular disease and related chronic diseases. Mt Sinai J Med 79: 632-640, 2012.

3. Muka T, Imo D, Jaspers L, Colpani V, Chaker L, van der Lee SJ, Mendis S, Chowdhury R, Bramer WM, Falla A, et al: The global impact of non-communicable diseases on healthcare spending and national income: A systematic review. Eur J Epidemiol 30: 251-277, 2015.

4. Guilherme A, Virbasius JV, Puri V and Czech MP: Adipocyte dysfunctions linking obesity to insulin resistance and type 2 diabetes. Nat Rev Mol Cell Biol 9: 367-377, 2008.

5. Xu H, Barnes GT, Yang Q, Tan G, Yang D, Chou CJ, Sole J, Nichols A, Ross JS, Tartaglia LA, et al: Chronic inflammation in fat plays a crucial role in the development of obesity-related insulin resistance. J Clin Invest 112: 1821-1830, 2003.

6. Ng M, Fleming T, Robinson M, Thomson B, Graetz N, Margono C, Mullany EC, Biryukov S, Abbafati C, Abera SF, et al: Global, regional, and national prevalence of overweight and obesity in children and adults during 1980-2013: A systematic analysis for the Global Burden of Disease Study 2013. Lancet 384: 766-781, 2014.

7. Whitman SC: A practical approach to using mice in atherosclerosis research. Clin Biochem Rev 25: 81-93, 2004.

8. Cannon CP, Harrington RA, James S, Ardissino D, Becker RC, Emanuelsson H, Husted S, Katus H, Keltai M, Khurmi NS, et al; PLATelet inhibition and patient outcomes investigators: Comparison of ticagrelor with clopidogrel in patients with a planned invasive strategy for acute coronary syndromes (PLATO): A randomised double-blind study. Lancet 375: 283-293, 2010.

9. Meier P and Timmis A: Almanac 2012: interventional cardiology: the national society journals present selected research that has driven recent advances in clinical cardiology. Heart 98: 1701-1709, 2012. 
10. Gómez-Gaviro MV, Lovell-Badge R, Fernández-Avilés F and Lara-Pezzi E: The vascular stem cell niche. J Cardiovasc Transl Res 5: 618-630, 2012.

11. Klein D, Weisshardt P, Kleff V, Jastrow H, Jakob HG and Ergün S: Vascular wall-resident CD $44^{+}$multipotent stem cells give rise to pericytes and smooth muscle cells and contribute to new vessel maturation. PLoS One 6: e20540, 2011.

12. Zengin E, Chalajour F, Gehling UM, Ito WD, Treede H, Lauke H, Weil J, Reichenspurner H, Kilic N and Ergün S: Vascular wall resident progenitor cells: A source for postnatal vasculogenesis. Development 133: 1543-1551, 2006.

13. Caplan AI: Mesenchymal stem cells. Stem Cells Transl Med 6: $1445-1451,2017$

14. Krampera M, Glennie S, Dyson J, Scott D, Laylor R, Simpson E and Dazzi F: Bone marrow mesenchymal stem cells inhibit the response of naive and memory antigen-specific $\mathrm{T}$ cells to their cognate peptide. Blood 101: 3722-3729, 2003.

15. Caplan AI: Adult mesenchymal stem cells for tissue engineering versus regenerative medicine. J Cell Physiol 213: 341-347, 2007.

16. Kode JA, Mukherjee S, Joglekar MV and Hardikar AA: Mesenchymal stem cells: Immunobiology and role in immunomodulation and tissue regeneration. Cytotherapy 11:377-391, 2009.

17. Prockop DJ, Brenner M, Fibbe WE, Horwitz E, Le Blanc K, Phinney DG, Simmons PJ, Sensebe L and Keating A: Defining the risks of mesenchymal stromal cell therapy. Cytotherapy 12 : 576-578, 2010

18. Tolar J, Le Blanc K, Keating A and Blazar BR: Concise review: Hitting the right spot with mesenchymal stromal cells. Stem Cells 28: 1446-1455, 2010.

19. Mirza A, Hyvelin JM, Rochefort GY, Lermusiaux P, Antier D, Awede B, Bonnet P, Domenech J and Eder V: Undifferentiated mesenchymal stem cells seeded on a vascular prosthesis contribute to the restoration of a physiologic vascular wall. J Vasc Surg 47: 1313-1321, 2008

20. Hashi CK, Zhu Y, Yang GY, Young WL, Hsiao BS, Wang K, Chu B and Li S: Antithrombogenic property of bone marrow mesenchymal stem cells in nanofibrous vascular grafts. Proc Natl Acad Sci USA 104: 11915-11920, 2007.

21. Gimble JM, Guilak F and Bunnell BA: Clinical and preclinical translation of cell-based therapies using adipose tissue-derived cells. Stem Cell Res Ther 1: 19, 2010.

22. De Ugarte DA, Morizono K, Elbarbary A, Alfonso Z, Zuk PA, Zhu M, Dragoo JL, Ashjian P, Thomas B, Benhaim P, et al: Comparison of multi-lineage cells from human adipose tissue and bone marrow. Cells Tissues Organs 174: 101-109, 2003.

23. Lee RH, Kim B, Choi I, Kim H, Choi HS, Suh K, Bae YC and Jung JS: Characterization and expression analysis of mesenchymal stem cells from human bone marrow and adipose tissue. Cell Physiol Biochem 14: 311-324, 2004.

24. Peng L, Jia Z, Yin X, Zhang X, Liu Y, Chen P, Ma K and Zhou C: Comparative analysis of mesenchymal stem cells from bone marrow, cartilage, and adipose tissue. Stem Cells Dev 17: 761-773, 2008

25. Zhang Y, Khan D, Delling $J$ and Tobiasch E: Mechanisms underlying the osteo- and adipo-differentiation of human mesenchymal stem cells. Sci World J 2012: 793823, 2012.

26. Mueller SM and Glowacki J: Age-related decline in the osteogenic potential of human bone marrow cells cultured in three-dimensional collagen sponges. J Cell Biochem 82: 583-590, 2001.

27. Stenderup K, Justesen J, Clausen C and Kassem M: Aging is associated with decreased maximal life span and accelerated senescence of bone marrow stromal cells. Bone 33: 919-926, 2003.

28. Kim Y,Kim H, Cho H, Bae Y, Suh K and Jung J: Direct comparison of human mesenchymal stem cells derived from adipose tissues and bone marrow in mediating neovascularization in response to vascular ischemia. Cell Physiol Biochem 20: 867-876, 2007.

29. van Steenberghe M, Schubert T, Guiot Y, Goebbels RM and Gianello P: Improvement of mesh recolonization in abdominal wall reconstruction with adipose vs. bone marrow mesenchymal stem cells in a rodent model. J Pediatr Surg 52: 1355-1362, 2017

30. Rasmussen JG, Frøbert O, Holst-Hansen C, Kastrup J, Baandrup U, Zachar V, Fink T and Simonsen U: Comparison of human adipose-derived stem cells and bone marrow-derived stem cells in a myocardial infarction model. Cell Transplant 23: 195-206, 2014

31. Razavi S, Zarkesh-Esfahani H, Morshed M, Vaezifar S, Karbasi S and GolozarMA: Nanobiocomposite of poly(lactide-co-glycolide)/ chitosan electrospun scaffold can promote proliferation and transdifferentiation of Schwann-like cells from human adiposederived stem cells. J Biomed Mater Res A 103: 2628-2634, 2015.
32. Kuhl C, Tautenhahn R, Böttcher C, Larson TR and Neumann S: CAMERA: An integrated strategy for compound spectra extraction and annotation of liquid chromatography/mass spectrometry data sets. Anal Chem 84: 283-289, 2012.

33. Trygg J, Holmes E and Lundstedt T: Chemometrics in metabonomics. J Proteome Res 6: 469-479, 2007.

34. van Velzen EJ, Westerhuis JA, van Duynhoven JP, van Dorsten FA, Hoefsloot HC, Jacobs DM, Smit S, Draijer R, Kroner CI and Smilde AK: Multilevel data analysis of a crossover designed human nutritional intervention study. J Proteome Res 7: 4483-4491, 2008.

35. Fong MY, McDunn J and Kakar SS: Identification of metabolites in the normal ovary and their transformation in primary and metastatic ovarian cancer. PLoS One 6: e19963, 2011.

36. Ke C, Hou Y, Zhang H, Yang K, Wang J, Guo B, Zhang F, Li H, Zhou X, Li Y, et al: Plasma Metabolic Profiles in Women are Menopause Dependent. PLoS One 10: e0141743, 2015.

37. Zhang T, Wu X, Yin M, Fan L, Zhang H, Zhao F, Zhang W, Ke C, Zhang G, Hou Y, et al: Discrimination between malignant and benign ovarian tumors by plasma metabolomic profiling using ultra performance liquid chromatography/mass spectrometry. Clin Chim Acta 413: 861-868, 2012.

38. Wishart DS, Jewison T, Guo AC, Wilson M, Knox C, Liu Y, Djoumbou Y, Mandal R, Aziat F, Dong E, et al: HMDB 3.0 - The Human Metabolome Database in 2013. Nucleic Acids Res 41: D801-D807, 2013.

39. Kanehisa M, Goto S, Sato Y, Kawashima M, Furumichi M and Tanabe M: Data, information, knowledge and principle: Back to metabolism in KEGG. Nucleic Acids Res 42: D199-D205, 2014.

40. No authors listed: Randomised trial of cholesterol lowering in 4444 patients with coronary heart disease: The Scandinavian Simvastatin Survival Study (4S). Lancet 344: 1383-1389, 1994.

41. Nordestgaard BG and Varbo A: Triglycerides and cardiovascular disease. Lancet 384: 626-635, 2014.

42. Rader DJ and Hovingh GK: HDL and cardiovascular disease. Lancet 384: 618-625, 2014.

43. Ridker PM: LDL cholesterol: Controversies and future therapeutic directions. Lancet 384: 607-617, 2014.

44. Campbell B and Kreider RB: Conjugated linoleic acids. Curr Sports Med Rep 7: 237-241, 2008.

45. Loscher CE, Draper E, Leavy O, Kelleher D, Mills KH and Roche HM: Conjugated linoleic acid suppresses NF-kappa B activation and IL-12 production in dendritic cells through ERK-mediated IL-10 induction. J Immunol 175: 4990-4998, 2005.

46. Kritchevsky D, Tepper SA, Wright S, Czarnecki SK, Wilson TA and Nicolosi RJ: Conjugated linoleic acid isomer effects in atherosclerosis: Growth and regression of lesions. Lipids 39: 611-616, 2004.

47. Toomey S, Harhen B, Roche HM, Fitzgerald D and Belton O: Profound resolution of early atherosclerosis with conjugated linoleic acid. Atherosclerosis 187: 40-49, 2006

48. Hassan Eftekhari M, Aliasghari F, Babaei-Beigi MA and Hasanzadeh J: Effect of conjugated linoleic acid and omega-3 fatty acid supplementation on inflammatory and oxidative stress markers in atherosclerotic patients. ARYA Atheroscler 9: 311-318, 2013.

49. Dilzer A and Park Y: Implication of conjugated linoleic acid (CLA) in human health. Crit Rev Food Sci Nutr 52: 488-513, 2012.

50. McCrorie TA, Keaveney EM, Wallace JM, Binns $\mathrm{N}$ and Livingstone MB: Human health effects of conjugated linoleic acid from milk and supplements. Nutr Res Rev 24: 206-227, 2011.

51. Onakpoya IJ, Posadzki PP, Watson LK, Davies LA and Ernst E: The efficacy of long-term conjugated linoleic acid (CLA) supplementation on body composition in overweight and obese individuals: A systematic review and meta-analysis of randomized clinical trials. Eur J Nutr 51: 127-134, 2012.

52. Risérus U, Berglund L and Vessby B: Conjugated linoleic acid (CLA) reduced abdominal adipose tissue in obese middle-aged men with signs of the metabolic syndrome: a randomised controlled trial. Int J Obes Relat Metab Disord 25: 1129-1135, 2001.

53. Churruca I, Fernández-Quintela A and Portillo MP: Conjugated linoleic acid isomers: Differences in metabolism and biological effects. Biofactors 35: 105-111, 2009.

54. Abduljabbar R, Al-Kaabi MM, Negm OH, Jerjees D, Muftah AA, Mukherjee A, Lai CF, Buluwela L, Ali S, Tighe PJ, et al: Prognostic and biological significance of peroxisome proliferator-activated receptor-gamma in luminal breast cancer. Breast Cancer Res Treat 150: 511-522, 2015. 
55. Huang WC, Tu RS, Chen YL, Tsai YY, Lin CF and Liou CJ: Conjugated linoleic acids suppress inflammatory response and ICAM-1 expression through inhibition of NF- $\mathrm{NB}$ and MAPK signaling in human bronchial epithelial cells. Food Funct 7: 2025-2033, 2016.

56. Smit LA, Baylin A and Campos $\mathrm{H}$ : Conjugated linoleic acid in adipose tissue and risk of myocardial infarction. Am J Clin Nutr 92: 34-40, 2010

57. Moloney F, Yeow TP, Mullen A, Nolan JJ and Roche HM Conjugated linoleic acid supplementation, insulin sensitivity, and lipoprotein metabolism in patients with type 2 diabetes mellitus. Am J Clin Nutr 80: 887-895, 2004.

58. Eyjolfson V, Spriet LL and Dyck DJ: Conjugated linoleic acid improves insulin sensitivity in young, sedentary humans. Med Sci Sports Exerc 36: 814-820, 2004.

59. Bassaganya-Riera J, Hontecillas R, Horne WT, Sandridge M, Herfarth HH, Bloomfeld R and Isaacs KL: Conjugated linoleic acid modulates immune responses in patients with mild to moderately active Crohn's disease. Clin Nutr 31: 721-727, 2012.

60. Larsson SC, Bergkvist L and Wolk A: High-fat dairy food and conjugated linoleic acid intakes in relation to colorectal cancer incidence in the Swedish Mammography Cohort. Am J Clin Nutr 82: 894-900, 2005.

61. McCann SE, Ip C, Ip MM, McGuire MK, Muti P, Edge SB Trevisan M and Freudenheim JL: Dietary intake of conjugated linoleic acids and risk of premenopausal and postmenopausal breast cancer, Western New York Exposures and Breast Cancer Study (WEB Study). Cancer Epidemiol Biomarkers Prev 13: 1480-1484, 2004.
62. Moon HS: Biological effects of conjugated linoleic acid on obesity-related cancers. Chem Biol Interact 224: 189-195, 2014.

63. Louw L: Effects of conjugated linoleic acid and high oleic acid safflower oil in the treatment of children with HPV-induced laryngeal papillomatosis: A randomized, double-blinded and crossover preliminary study. Lipids Health Dis 11: 136, 2012.

64. Liu GY, Liu J, Wang YL, Liu Y, Shao Y, Han Y, Qin YR, Xiao FJ, Li PF, Zhao LJ, et al: Adipose-derived mesenchymal stem cells ameliorate lipid metabolic disturbance in mice. Stem Cells Transl Med 5: 1162-1170, 2016.

65. Martins SV, Madeira A, Lopes PA, Pires VM, Alfaia CM, Prates JA, Moura T and Soveral G: Adipocyte membrane glycerol permeability is involved in the anti-adipogenic effect of conjugated linoleic acid. Biochem Biophys Res Commun 458: 356-361, 2015.

66. Ivanova-Todorova E, Bochev I, Mourdjeva M, Dimitrov R, Bukarev D, Kyurkchiev S, Tivchev P, Altunkova I and Kyurkchiev DS: Adipose tissue-derived mesenchymal stem cells are more potent suppressors of dendritic cells differentiation compared to bone marrow-derived mesenchymal stem cells. Immunol Lett 126: 37-42, 2009.

(i) $\Theta$ This work is licensed under a Creative Commons Attribution-NonCommercial-NoDerivatives 4.0 International (CC BY-NC-ND 4.0) License. 